\title{
Risk stratification and outreach to hematology/oncology patients during the COVID-19 pandemic
}

\author{
Chelsea K. Osterman ${ }^{1}$ (D) - Tammy Triglianos ${ }^{1}$ - Gary S. Winzelberg ${ }^{2} \cdot$ Angela D. Nichols $^{3}$ • Julia Rodriguez-O'Donnell ${ }^{4}$. \\ Sharon M. Bigelow ${ }^{5}$ Hendrik van Deventer ${ }^{3,5} \cdot$ Hanna K. Sanoff ${ }^{1,5} \cdot$ Emily M. Ray ${ }^{1,5}$
}

Received: 9 July 2020 / Accepted: 4 September 2020 / Published online: 12 October 2020

(C) Springer-Verlag GmbH Germany, part of Springer Nature 2020

\begin{abstract}
Purpose Cancer patients have many medical and psychosocial needs, which may increase during the COVID-19 pandemic. We sought to (1) risk-stratify hematology/oncology patients using general medicine and cancer-specific methods to identify those at high risk for acute care utilization, (2) measure the correlation between two risk stratification methods, and (3) perform a telephone-based needs assessment with intervention for high-risk patients.

Methods Patients were risk-stratified using a general medical health composite score (HCS) and a cancer-specific risk (CSR) stratification based on disease and treatment characteristics. The correlation between HCS and CSR was measured using Spearman's correlation. A multi-disciplinary team developed a focused needs assessment script with recommended interventions for patients categorized as high-risk by either method. The number of patient needs identified and referrals for services made in the first month of outreach are reported.

Results A total of 1697 patients were risk-stratified, with 17\% high-risk using HCS and 22\% high-risk using CSR. Correlation between HCS and CSR was modest $(\rho=0.41)$. During the first month of the pilot, 286 patients were called for outreach with 245 contacted (86\%). Commonly identified needs were financial difficulties (17\%), uncontrolled symptoms (15\%), and interest in advance care planning (13\%), resulting in referral for supportive services for $33 \%$ of patients.

Conclusion There is a high burden of unmet medical and psychosocial needs in hematology/oncology patients during the COVID-19 pandemic. A telephone-based outreach program results in the identification of and intervention for these needs; however, additional cancer-specific risk models are needed to improve targeting to high-risk patients.
\end{abstract}

Keywords Risk assessment $\cdot$ Coronavirus $\cdot$ COVID-19 $\cdot$ Malignancy $\cdot$ Quality improvement

Electronic supplementary material The online version of this article (https://doi.org/10.1007/s00520-020-05744-y) contains supplementary material, which is available to authorized users.

Chelsea K. Osterman

Chelsea.osterman@unchealth.unc.edu

1 Division of Oncology, Department of Medicine, University of North Carolina, 170 Manning Drive, CB 7305, Chapel Hill, NC 27599, USA

2 Division of Geriatric Medicine, Department of Medicine, University of North Carolina, Chapel Hill, NC, USA

3 Division of Hematology, Department of Medicine, University of North Carolina, Chapel Hill, NC, USA

4 Department of Social Work, University of North Carolina, Chapel Hill, NC, USA

5 Lineberger Comprehensive Cancer Center, University of North Carolina, Chapel Hill, NC, USA

\section{Introduction}

Due to the high risk for contracting and suffering complications of COVID-19 among oncology patients, prevention of acute healthcare utilization, such as emergency room (ER) visits and hospitalizations, is increasingly important during the COVID-19 pandemic [1,2]. The pandemic has forced rapid changes to healthcare delivery, including increased utilization of telemedicine, which may introduce new barriers to care and new challenges to systematically addressing patient concerns, in turn increasing the risk for acute healthcare utilization.

Oncology navigation programs and proactive symptom management can decrease ER visits and hospitalizations [3-5]. However, due to resource constraints, risk stratification is needed to prioritize patients for interventions. The applicability of general medical risk stratification tools to oncology 
patients is unclear, and few cancer-specific models exist. We sought to (1) risk-stratify oncology patients using a general medicine Health Composite Score (HCS) and a cancerspecific risk (CSR) stratification to identify those at high risk for acute healthcare utilization, (2) measure the correlation between risk stratification methods, and (3) pilot implementation of a telephone-based outreach for high-risk patients.

\section{Methods}

\section{Risk stratification}

Given the need to risk stratify patients expediently, we explored whether a pre-existing HCS could be applied. The HCS was developed at the University of North Carolina (UNC) and is predictive of 90-day hospitalization in a general medicine population [6]. The HCS is calculated within the electronic medical record (EMR) and incorporates information from UNC and other healthcare systems using the same EMR. It includes inpatient admissions and ER visits in the past year, active medications, unique providers seen in the past year, high-impact chronic conditions, uncontrolled chronic diseases, inpatient psychiatry admission in the past 5 years, age, insurance, and primary care provider status. Each component is weighted to provide a total score of $0-100$, with patients categorized as low $(0-8)$, moderate $(9-20)$, or high risk (21-100).

Our CSR stratification was adapted from the model developed by Rocque and colleagues [3]. For the CSR, solid tumor patients with an ER visit or hospitalization in the past month, active aggressive treatment, or advanced malignancy with high symptom burden were high risk; those receiving moderately immunosuppressive or low-intensity therapies with multiple comorbidities were moderate risk; and patients on hormonal therapy or long-term survivors were low risk. Patients with hematologic malignancies receiving inpatient or highrisk therapy or transfusion were high risk; those on lower risk multi-agent therapy, newly started on single-agent therapy, or recently completing therapy were moderate risk; and longterm survivors and patients on single-agent long-term oral therapies were low risk. For this pilot, the HCS and CSR were applied to the patients of 16 providers, creating a sample representative of the major cancer types. The association between HCS and CSR scores was evaluated using Spearman's correlation.

\section{Outreach}

We developed a telephone script and EMR template with input from hematology/oncology, nurse navigation, palliative care, psychiatry, social work, and the patient and family resource center. The script emphasized that care teams remain available and included directed questions regarding symptoms, medications, coping, resources, advanced care planning (ACP), and cancer care. The template outlined interventions for identified needs, including referral to social work, palliative care, and the patient and family resource center, which includes staff and volunteers to provide emotional support and direct patients to additional resources. It concluded with a summary of active issues, interventions made, and time frame for follow-up. During the pilot, the template was iteratively revised to improve clarity and functionality based on caller feedback (Online Resource 1). Outreach began with patients identified as high risk by either HCS or CSR. To assess the initial impact of our outreach, we measured the number of patient needs identified and interventions made in the first month.

\section{Results}

\section{Risk stratification}

A total of 1697 patients were risk-stratified (Online Resource 2). By HCS, $17 \%, 49 \%$, and $34 \%$ of patients were high, moderate, and low risk, respectively. By CSR, $22 \%, 37 \%$, and $41 \%$ of patients were high, moderate, and low risk, respectively. The correlation between HCS and CSR was moderate at 0.41 .

\section{Outreach}

During the first month of outreach, 286 patients were called with $245(86 \%)$ successfully contacted. Most calls were made by nurse navigators $(66 \%)$, with a mean call duration of 14 min. Commonly identified patient issues were financial difficulties $(17 \%)$, uncontrolled symptoms $(15 \%)$, and interest in ACP $(13 \%)$ (Table 1). Overall, 33\% of patients were referred for additional services (Online Resource 3).

\section{Discussion}

Reducing acute healthcare utilization requires the identification of high-risk patients, and few cancer-specific risk stratification models exist [7]. In this pilot, we found that a general medicine composite score has only modest correlation with a cancer-specific risk stratification, failing to capture risks associated with a cancer diagnosis and treatment. The refinement of our CSR model is ongoing, and the final model will be evaluated to assess its ability to predict acute healthcare utilization among oncology patients. The development of such a tool is increasingly important, even beyond the pandemic, as provider-payer partnerships seek to improve the quality of cancer care while decreasing costs $[8,9]$. 
Table 1 Patient needs identified during outreach phone calls, no. (\%)

\begin{tabular}{ll}
\hline Issue or need identified & Patients $(n=245)$ \\
\hline Financial difficulties & $42(17)$ \\
Uncontrolled symptoms & $36(15)$ \\
Interest in advance directives or healthcare power of attorney & $31(13)$ \\
Medication issues (i.e., refills, questions, compliance, cost) & $31(13)$ \\
Uncertain how to contact the care team & $24(10)$ \\
Question about the plan of care & $17(7)$ \\
Unaware of the next oncology appointment & $17(7)$ \\
Distress score $\geq 8 / 10$ & $14(6)$ \\
No future appointment scheduled or schedule is incorrect & $7(3)$ \\
\hline
\end{tabular}

Importantly, despite a robust nurse navigation program available to all our oncology patients, $33 \%$ of patients had additional needs uncovered through outreach during the COVID-19 pandemic. This may reflect the nature of the questions included in the outreach, which are not all routinely asked in clinical encounters, as well as new patient needs emerging during the pandemic. A protocol for interventions resulted in a high rate of referrals to address these needs.

Our pilot was implemented at a well-resourced academic center and may have limited generalizability to lowerresourced settings. Our ongoing efforts to develop an automated EMR-based stratification system and enlist lay navigators for outreach could create a more sustainable program. Continued efforts to identify high-risk oncology patients and address their needs are warranted both during and after the pandemic in order to decrease acute healthcare utilization and improve care quality.

\section{Conclusions}

This pilot demonstrates a high burden of unmet medical and psychosocial needs in oncology patients during the COVID19 pandemic and provides a framework for systematically addressing these needs through risk stratification and outreach.

Authors' contributions All authors contributed to the study conception and design. Material preparation, data collection, and analysis were performed by Chelsea Osterman and Emily Ray. The first draft of the manuscript was written by Chelsea Osterman and all authors commented on previous versions of the manuscript. All authors read and approved the final manuscript.

Funding Dr. Osterman is supported by a National Research Service Award Post-Doctoral Traineeship from the Agency for Healthcare Research and Quality sponsored by The Cecil G. Sheps Center for Health Services Research, The University of North Carolina at Chapel Hill (grant 5T32H2000032).

Data availability Original data is available to researchers upon request.

\section{Compliance with ethical standards}

Conflict of interest Dr. Osterman has received grant support from the Agency for Healthcare Research and Quality. Dr. Sanoff has received grant support outside of this work from the Patient-Centered Outcomes Research Institute. Dr. Ray has received grant support outside of this work from the Conquer Cancer Foundation/Breast Cancer Research Fund, Agency for Healthcare Research and Quality, and Lung Cancer Initiative of North Carolina.

Ethics approval This is a quality improvement study. The Office of Human Research Ethics at the University of Carolina has confirmed that Institutional Review Board approval is not required (IRB 20-1090).

Consent to participate Not applicable.

Consent for publication Not applicable.

Code availability Not applicable.

\section{References}

1. Liang W, Guan W, Chen R, Wang W, Li J, Xu K, Li C, Ai Q, Lu W, Liang H, Li S, He J (2020) Cancer patients in SARS-CoV-2 infection: a nationwide analysis in China. Lancet Oncol 21:335-337. https://doi.org/10.1016/S1470-2045(20)30096-6

2. He W, Chen L, Chen L, Yuan G, Fang Y, Chen W, Wu D, Liang B, Lu X, Ma Y, Li L, Wang H, Chen Z, Li Q, Gale RP (2020) COVID19 in persons with haematological cancers. Leukemia. 34:16371645. https://doi.org/10.1038/s41375-020-0836-7

3. Rocque GB, Pisu M, Jackson BE, Kvale EA, Demark-Wahnefried W, Martin MY, Meneses K, Li Y, Taylor RA, Acemgil A, Williams CP, Lisovicz N, Fouad M, Kenzik KM, Partridge EE, for the Patient Care Connect Group (2017) Resource use and medicare costs during lay navigation for geriatric patients with cancer. JAMA Oncol 3: 817-825. https://doi.org/10.1001/jamaoncol.2016.6307

4. Rocque GB, Partridge EE, Pisu M, Martin MY, Demark-Wahnefried W, Acemgil A, Kenzik K, Kvale EA, Meneses K, Li X, Li Y, Halilova KI, Jackson BE, Chambless C, Lisovicz N, Fouad M, Taylor RA (2016) The Patient Care Connect Program: transforming health care through lay navigation. J Oncol Pract 126:e633-e642. https://doi.org/10.1200/jop.2015.008896

5. Barkley R, Soobader M-J, Wang J, Blau S, Page RD (2019) Reducing cancer costs through symptom management and triage 
pathways. J Oncol Pract 15:e91-e97. https://doi.org/10.1200/jop.18. 00082

6. Jarmul JA, Whitney A, Gwynne M et al (2017) Validation of a composite health score to predict 90-day all-cause hospitalization in a population of general medicine and family medicine patients seen at two large, academic faculty practices. J Gen Intern Med 32(Suppl 2)

7. Handley NR, Schuchter LM, Bekelman JE (2018) Best Practices for Reducing Unplanned Acute Care for Patients With Cancer. J Oncol Pract 14:306-313. https://doi.org/10.1200/jop.17.00081

8. Aviki EM, Schleicher SM, Mullangi S (2019) The oncology care model and other value-based payment models in cancer care.
JAMA Oncol 5:298-299. https://doi.org/10.1001/jamaoncol.2018. 5735

9. Ward JC, Bourbeau BR, Chin AL, Page RD, Grubbs SS, Kamin DY, Green SR, Rappaport M (2020) Updates to the ASCO patientcentered oncology payment model. J Oncol Pract 16:263-269. https://doi.org/10.1200/jop.19.00776

Publisher's note Springer Nature remains neutral with regard to jurisdictional claims in published maps and institutional affiliations. 